An official journal of the / Un journal officiel de la "Société Sénégalaise de Cancérologie" (SOSECAN)

Journal homepage: www.africanjournalofoncology.com

Editorial / Editoriale

DOI: https://www.doi.org/I0.54266/ajo.I.2.3I.EPJF6430

\title{
Become stronger through diversity
}

\section{Devenir plus fort à travers la diversité}

S. Ka ${ }^{\prime *}$, MM. Dieng', ME. Charfi', A. Dem'.

' Institut Joliot Curie de Dakar, Sénégal.

La cancérologie est une discipline multidisciplinaire. Ne dit-on pas : " malheur à celui qui se veut cancérologue tout seul?". Autant la chirurgie est traditionnellement considérée comme la pierre angulaire du traitement des cancers solides, autant les traitements médicaux des cancers et la radiothérapie, au-delà de leurs caractères néo-adjuvants ou adjuvants, indispensables pour assurer une meilleure prise en charge des cancers, tendent de plus en plus à repousser les frontières des intervalles de survie [I, 2].

Certains cancers solides sont connus depuis des décennies comme curables par la seule chimiothérapie. Les exemples en sont les tumeurs embryonnaires et les tumeurs de l'enfant dont les formes métastatiques peuvent être guéries jusqu'à l'ordre de $80 \%$ [3]. D'autres sont traités exclusivement par la radiothérapie ou par le couple potentialisateur que constitue la radio-chimiothérapie. Comme c'est le cas des cancers du col de l'utérus ou ORL [4].

En Afrique, dans un contexte de ressources modestes, on retrouve 2 situations morbides particulières que sont la rareté de la ressource humaine qualifiée dans le traitement des cancers et les limites de l'organisation des systèmes [5].

Peu de pôles académiques proposent la formation de spécialistes dans les différents domaines de la cancérologie et peu de centres favorisent la synergie des actions des différents praticiens. Comme en atteste la rareté des réunions de concertations pluridisciplinaires (RCP) dont l'impact positif est établi [6].

Les outils de vulgarisation de la cancérologie notamment les journaux spécialisés doivent s'ouvrir à ces différents aspects et participer à la promotion des bonnes pratiques en cancérologie.

Cette étape de la vie du Journal Africain d'Oncologie coïncide avec la plus vaste campagne de vaccination jamais entreprise par l'humanité. Des interférences manifestes existent entre les soins en cancérologie et la prise en charge des patients dans ce contexte de pandémie à la Covid-19. D'où la nécessaire publication des recommandations pour la vaccination chez les patients en cancérologie.

Le Journal Africain d'Oncologie à travers la diversité de son comité éditorial participera en âme et conscience, avec la vérité scientifique ou ce qui s'en rapproche le plus à l'état actuel des connaissances et des moyens disponibles en bandoulière, à relever les niveaux de connaissances en cancérologie et à mettre au même niveau d'informations les formateurs, les praticiens, les décideurs et les populations.

\section{CONFLITS D'INTERET}

Les auteurs n'ont déclaré aucun conflit d'intérêts.

\section{REFERENCES}

I. Kemeny M. Surgical oncology. Am J Surg. 2007; 193(I): 133-4.

2. Popescu RA, Schäfer R, Califano R, Eckert R, Coleman $R$, Douillard JY et al. The current and future role of the medical oncologist in the professional care for cancer patients: a position paper by the European Society for Medical Oncology (ESMO). Ann Oncol. $2014 ; 25(\mathrm{I}): 9-15$.

3. Adra N, Einhorn LH. Testicular cancer update. Clin Adv Hematol Oncol. 20I7; I5(5):386-396.

4. Naga Ch P, Gurram L, Chopra S, Mahantshetty U. The management of locally advanced cervical cancer. Curr Opin Oncol. 2018;30(5):323-329.

5. Mutebi M, Adewole I, Orem J, Abdella K, Coker O, Kolawole I et al. African Cancer Coalition. Toward Optimization of Cancer Care in Sub-Saharan Africa: Development of National Comprehensive Cancer Network Harmonized Guidelines for Sub-Saharan Africa. JCO Glob Oncol. 2020 Sep;6:14I2-1418.

6. Brandão $M$, Guisseve A, Bata G, Firmino-Machado J, Alberto M, Ferro J et al. Survival Impact and CostEffectiveness of a Multidisciplinary Tumor Board for Breast Cancer in Mozambique, Sub-Saharan Africa. Oncologist. 2021;26(6):e996-el 008. 